\title{
Note on the $\mathrm{R}^{2}$ measure of goodness of fit for nonlinear models
}

\author{
TARALD O. KVÅLSETH \\ University of Minnesota, Minneapolis, Minnesota 55455
}

\begin{abstract}
The coefficient of determination $\mathrm{R}^{2}$ is a standard measure of goodness of fit for mathematical models fitted to empirical data by means of least squares regression. However, for the case of nonlinear models, such as power models and exponential models frequently used in the behav. ioral sciences, the $\mathbf{R}^{2}$ measure is often subject to incorrect calculations and misinterpretations, producing potentially misleading results. This paper discusses these $R^{2}$-related issues and presents the proper method of calculation. A fictitious example is used.
\end{abstract}

The purpose of this note is to point out some not so obvious mistakes that are frequently made when using the coefficient of determination $\mathrm{R}^{2}$ as a measure of the goodness of fit for nonlinear models such as

$$
\hat{\mathrm{Y}}=\mathrm{a} \mathrm{X}^{\mathrm{b}} ; \hat{\mathrm{Y}}=\mathrm{a} \mathrm{e}^{\mathrm{bX}},
$$

where $\hat{Y}$ refers to the fitted (calculated) values of the dependent variable as opposed to its actual (observed) values, Y (no subscripts will be used in this paper for notational simplicity). Of course, the determination of the parameter estimates $a$ and $b$ by means of the standard method of ordinary linear least squares regression requires a transformation of the models in Equation 1 to a general linear model of the form

$$
\hat{Y}=a+b X
$$

by taking logarithms of both sides of the equalities in Equation 1.

The explanatory power of such fitted models in terms of the proportion of the variation in the dependent variable "explained" or "accounted for" by the fitted models is measured by the $\mathrm{R}^{2}$ and for which the following three expressions are used interchangeably in the literature:

$$
\begin{gathered}
\mathrm{R}_{1}^{2}=1-\left[\Sigma(\mathrm{Y}-\hat{\mathrm{Y}})^{2} / \Sigma(\mathrm{Y}-\overline{\mathrm{Y}})^{2}\right], \\
\mathrm{R}_{2}^{2}=\Sigma(\hat{\mathrm{Y}}-\overline{\mathrm{Y}})^{2} / \Sigma(\mathrm{Y}-\overline{\mathrm{Y}})^{2},
\end{gathered}
$$

and

$$
\mathrm{R}_{3}^{2}=\Sigma(\hat{\mathrm{Y}}-\overline{\hat{\mathrm{Y}}})^{2} / \Sigma(\mathrm{Y}-\overline{\mathrm{Y}})^{2},
$$

where $\bar{Y}$ and $\overline{\hat{Y}}$ denote the sample mean of the actual and fitted $\mathrm{Y}$ values, respectively. A related measure of

Requests for reprints should be addressed to T. O. Kvålseth, Department of Mechanical Engineering, University of Minnesota, Minneapolis, Minnesota 55455. goodness of fit frequently used is the standard error of prediction $s_{e}$ given by

$$
s_{e}=\sqrt{\Sigma(Y-\hat{Y})^{2} /(N-2)}
$$

and

$$
s_{e}=\sqrt{\left(1-R_{1}^{2}\right) \Sigma(Y-\bar{Y})^{2} /(N-2)},
$$

where the last equality follows from Equation 3 and where $\mathrm{N}$ denotes the number of $\mathrm{Y}$ (and $\mathrm{X}$ ) values in the data sample.

It is perfectly appropriate to use any one of the $\mathbf{R}^{2}$ expressions in Equations 3-5 for the case of a general linear model, as in Equation 2 (and also for multivariate linear models), for which it can be easily shown that $R_{1}^{2}=R_{2}^{2}=R_{3}^{2}$ (e.g., Goldberger, 1964, Chapter 4). However, this is not so for the nonlinear models in Equation 1, a fact that does not appear to be properly recognized. Furthermore, for such nonlinear models, both $R_{2}^{2}$ and $R_{3}^{2}$ may possibly be larger than 1 , whereas it is obvious from Equation 3 that $R_{1}^{2} \leqslant 1$ always. As an exemplification of such $\mathbf{R}^{2}$ differences, consider the following data:

$\begin{array}{rrrrrrr}X & 1 & 2 & 3 & 4 & 5 & 6 \\ Y & 15 & 37 & 52 & 59 & 83 & 92\end{array}$

When the power model in Equation 1 is fitted to these data, the following $R^{2}$ values are obtained: $R_{1}^{2}=.9777$, $R_{2}^{2}=1.0984$, and $R_{3}^{2}=1.0983$. Note also in passing that for the case of the linear model in Equation 2, the linear correlation coefficient $r_{\hat{Y}} Y$ between $\hat{Y}$ and $Y$ is such that $r_{\hat{Y} \mathbf{Y}}^{2}=R_{1}^{2}=R_{2}^{2}=R_{3}^{2}$ (e.g., Draper \& Smith, 1981), whereas this is not so for the nonlinear models in Equation 1, with $\mathrm{r}_{\hat{\mathrm{Y}} \mathrm{Y}}^{2}=.9811$ for the power model fitted to the preceding data.

The question is, then, which one of the $\mathrm{R}^{2}$ expressions in Equations 3-5 is the appropriate one for nonlinear models such as those in Equation 1? In order to 
have always $R^{2} \leqslant 1$, the expression in Equation 3 is the obvious choice, which is then appropriate for both linear and nonlinear models. ${ }^{1}$ If a model specification is not entirely incompatible with given data, the expression in Equation 3 will also yield $R^{2} \geqslant 0$. For this $R^{2}$ expression, with $\Sigma(\mathrm{Y}-\hat{\mathrm{Y}})^{2}$ measuring the variation of $\mathrm{Y}$ about the fitted model, the unexplained variation, $100 R^{2}$, measures the percentage of the $Y$ variation explained by the fitted model.

Another $\mathrm{R}^{2}$-related mistake frequently made for the nonlinear models in Equation 1 is to use the transformed regressand $\log Y$ when calculating $\mathrm{R}^{2}$ according to one of the expressions in Equations 3-5, or equivalently, according to the square of the linear correlation coefficient between $\log \mathrm{Y}$ and $\log \hat{\mathrm{Y}}$. Such incorrect $\mathrm{R}^{2}$ calculation is incorporated, for example, in the curve-fitting programs for some programmable calculators (HewlettPackard, 1976; Texas Instruments, 1977). ${ }^{2}$ The $\mathrm{R}^{2}$ calculation based on $\log Y$ and $\log \hat{Y}$ provides a measure of the goodness of fit of the model $\log \hat{Y}=\log a+b \log X$ or $\log Y=\log a+b X$ in terms of the proportion of the variation in $\log \mathrm{Y}$ accounted for, which is different from the proportion of the variation in $Y$ accounted for and as measured by the formula in Equation 3 for the models in Equation 1. Thus, for example, when comparing the fits of a power model and a linear model to the same set of data, misleading results may be obtained when the two $R^{2}$ s are on an incomparable footing by using $\log Y$ in the $R^{2}$ calculation for the power model and $\mathrm{Y}$ in $\mathrm{R}^{2}$ for the linear model. An indication of this fact is provided by the $\mathrm{X}$ and $\mathrm{Y}$ data given above and for which it is found that $\mathrm{R}^{2}=.9808$ for the linear model $\hat{\mathrm{Y}}=\mathrm{a}+\mathrm{bX}$ and $\mathrm{R}^{2}=.9816$ for $\log \hat{\mathrm{Y}}=\log \mathrm{a}+\mathrm{b} \log \mathrm{X}$. These two $R^{2}$ values may then be incorrectly interpreted by some as implying a slight preference for the power model $\hat{\mathrm{Y}}=\mathrm{aX}^{\mathrm{b}}$, whereas the linear model $\hat{\mathrm{Y}}=$ $a+b X$ does in fact provide a fit superior to that of the power model for which the correct $R^{2}$ value from Equation 3 is .9777 , as mentioned above.
In conclusion, it may be pointed out that while the above arguments have focused on models with one independent variable $(\mathrm{X})$, they are also directly applicable to multivariate nonlinear models, with some obvious modifications. The arguments are also relevant to nonlinear models other than those of Equation 1.

\section{REFERENCES}

Draper, N. R., \& Smith, H. Applied regression analysis (2nd ed.). New York: Wiley, 1981.

Goldberger, A. S. Econometric theory. New York: Wiley, 1964. HeWLETT-PACKARD. HP-67 standard pack. Corvallis, Ore: Author, 1976.

TeXas Instruments. TI programmable 58/59 applied statistics. Austin, Tex: Author, 1977.

\section{NOTES}

1. Another $\mathrm{R}^{2}$ expression that is also sometimes being used is

$$
\mathrm{R}_{4}^{2}=1-\left[\Sigma(\hat{\mathrm{e}}-\overline{\mathrm{e}})^{2} / \Sigma(\mathrm{Y}-\overline{\mathrm{Y}})^{2}\right] ; \hat{\mathrm{e}}=\mathrm{Y}-\hat{\mathrm{Y}},
$$

where $R_{4}^{2} \geqslant R_{1}^{2}$ and with equality if and only if $\bar{Y}=\overline{\hat{Y}}$. For the linear model in Equation 2, and also for general multivariate linear (in the parameters) models, it can easily be shown that $\overline{\mathrm{Y}}=\hat{\hat{Y}}$ so that $R_{4}^{2}=R_{1}^{2}=R_{2}^{2}=R_{3}^{2}$. However, for the nonlinear models in Equation 1, $R_{4}^{2}>R_{1}^{2}$ with, for example, $R_{4}^{2}=.9778$ for the power model in Equation 1 fitted to the above $\mathrm{X}$ and $\mathrm{Y}$ data.

2. For the power model, for example, the HP67/97 program calculates $\mathrm{R}^{2}$ as the square of the linear correlation coefficient between $\log \mathrm{Y}$ and $\log \mathrm{X}$, which is equivalent to the square of the linear correlation coefficient between $\log Y$ and $\log \hat{Y}$; the TI 58/ 59 program calculates (the unsquared) $R$ in the same way.

Steven J. Hansen has made an extension to the HP67/97 curve-fitting program for calculating $\mathrm{R}^{2}$ according to Equation 3 and $\mathrm{s}_{\mathrm{e}}$ according to Equation 6 for the power model, which is also applicable to the exponential model, since the exponential model may, of course, be treated as a power model in which $\hat{\mathbf{Y}}=\mathbf{a} \mathrm{e}^{\mathbf{b X}}=\mathrm{a}\left(\mathrm{e}^{\mathbf{X}}\right)^{\mathbf{b}}=\mathbf{a} \mathrm{Z}^{\mathbf{b}}$. Copies of the program listings are available from the author (T.O.K.).

(Received for publication December 20, 1982.) 